\title{
Die Tauglichkeit des Quotenverfahrensschemas unter Urnenmodellbedingungen *
}

\author{
Andreas Quatember \\ Institut für Angewandte Statistik, Universität Linz
}

\begin{abstract}
Zusammenfassung: Unter „Urnenmodellbedingungen“, also bei gleichen Auswahlwahrscheinlichkeiten für alle Objekte der Grundgesamtheit und ohne Berücksichtigung der Nichtstichprobenfehler, entspricht die Auswahl nach kombinierten Quoten einer nach einem mehrdimensionalen Merkmal geschichteten Zufallsauswahl. Sie kann daher auch die statistischen Eigenschaften dieses Verfahrens, die Erwartungstreue des damit erhobenen Schätzers für den Parameter $\mu$ des Untersuchungsmerkmals und den Genauigkeitsgewinn im Vergleich zu einer uneingeschränkten Zufallsauswahl für sich beanspruchen. Die Auswahl nach Randquoten liefert im allgemeinen selbst unter diesen Annahmen verzerrte Schätzungen für $\mu$. Es lassen sich jedoch Bedingungen hinsichtlich der Quotenmerkmale angeben, unter denen dennoch erwartungstreue Schätzungen erzielt werden können und daraus werden Vorschläge für die Praktiker abgeleitet.
\end{abstract}

\begin{abstract}
Under "urnmodel-conditions", this means by using equal inclusion probabilities for all objects in the population and without consideration of the nonsampling-error, quota sampling with combined quotas is stratified random sampling with a multidimensional stratificationvariable. Therefore it can claim the statistical attributes of stratified sampling, the unbiasedness of its estimators for the parameter under interest $\mu$ and the gain in precision compared to simple random sampling. In general - even under these assumptions - quota sampling with marginal quotas produces biased estimators for $\mu$. The conditions for an unbiased estimation concerning the quota variables are determined and so proposals for the practical use of quota sampling are derived.
\end{abstract}

Schlüsselwörter: Stichprobenverfahren, Quotenverfahren, Markt- und Meinungsforschung

\section{Einleitung}

Das Quotenverfahren ist das in der kommerziellen Markt- und Meinungsforschung am häufigsten verwendete Auswahlverfahren zur Ziehung von Stichproben. Laut Auskunft führender österreichischer Markt- und Meinungsforschungsinstitute werden derzeit in

\footnotetext{
* Schriftliche ausführliche Ausarbeitung eines Vortrages, gehalten an den „Österreichischen Statistik-Tagen 1997“ der Österreichischen Statistischen Gesellschaft in Wien.
} 
Österreich etwa 70\% aller Umfragen mit diesem Verfahren durchgeführt. In seiner Konzeption weist es starke Ähnlichkeiten zum Schichtenverfahren mit proportionaler Allokation des Stichprobenumfanges auf die Schichten auf. Die konkrete Auswahl der Zielpersonen innerhalb der Schichten erfolgt jedoch nicht wie bei diesem uneingeschränkt zufällig, sondern liegt im Ermessen des Interviewers.

Dieser Umstand ist die Ursache dafür, daß die statistischen Eigenschaften der damit erhobenen Schätzer in der Standardliteratur über Stichprobenverfahren, sofern diese Auswahltechnik überhaupt Erwähnung findet, nicht untersucht werden. Dies ist insofern bemerkenswert, als bei den sogenannten Zufallsauswahlen - im Gegensatz dazu - gerade so getan wird, als ob der in der klassischen Stichprobentheorie nicht behandelte Nichtstichprobenfehler, also jener Teil der Abweichung einer Statistik von ihrem Parameter, der nicht durch den Umstand der Ziehung einer Stichprobe erklärt werden kann, bei diesen gar nicht existierte. Ein Nichtstichprobenfehler wird etwa hervorgerufen durch das Nichtantreffen der Zielpersonen oder deren Verweigerung des gesamten Interviews, bestimmter oder richtiger Auskünfte. Im Vergleich zu den Zufallsauswahlen besitzt das Quotenverfahren diesbezüglich aber lediglich eine zusätzliche Fehlerquelle: die Interviewerfreiheit bei der Auswahl der Zielpersonen. Da es auch noch das erklärte Ziel

- der von den Markt- und Meinungsforschungsinstituten vorgenommenen zentralen Verwaltung des Interviewerstabes,

- der Schulung der Interviewer und deren Kontrolle,

- der geringen Anzahl von Befragungspersonen pro Interviewer und

- einer Verteilung der Stichprobe auf ausgewählten Merkmalen, die der Verteilung der Grundgesamtheit auf diesen Merkmalen entspricht,

ist, diese Freiheit einzuschränken (vgl. etwa Schmidtchen, 1961, S. 37ff), stellt sich die Frage, was das Quotenverfahrensschema unter Urnenmodellbedingungen, also bei zufälliger Ziehung der Objekte für die Stichprobe aus der Grundgesamtheit und ohne Berücksichtigung des Nichtstichprobenfehlers, zu leisten imstande ist.

\section{Die Quotenauswahlverfahren}

Bei Wahl bestimmter Merkmale (z.B. Alter und Geschlecht) als Quotenmerkmale hat sich die Stichprobe mit den gleichen Anteilen, den sogenannten Quoten, wie die Grundgesamtheit auf diesen Merkmalen zu verteilen. Die Quoten für diese Merkmale können dabei kombiniert (z.B. bei Verwendung von Alter und Geschlecht: Wieviele Frauen einer bestimmten Altersklasse, wieviele Männer dieser Altersklasse, wieviele Frauen einer anderen Altersklasse usf. zu befragen sind) oder getrennt (z.B. wieviele Frauen und wieviele Männer und wieviele Personen aus den verschiedenen Altersklassen zu befragen sind) vorgegeben werden. Im einen Fall spricht man dann von einer Auswahl nach kombinierten Quoten, im anderen von einer Auswahl nach Randquoten.

Was die Herkunft des Quotenverfahrens betrifft, führt die augenscheinliche Ähnlichkeit zum Schichtenverfahren jedoch in die Irre. Denn das Quotenverfahren ist nicht aus dieser bereits in den Zwanzigerjahren unseren Jahrhunderts angewendeten und 
von Jerzy Neyman 1934 vollständig entwickelten theoretischen Konzeption (vgl. Bellhouse, 1988, S. 7ff) entstanden, sondern aus den Bedürfnissen der sich in den Dreißigerjahren in den Vereinigten Staaten von Amerika entwickelnden kommerziellen Markt- und Meinungsforschung. Die Verwendung von Quotenmerkmalen basiert demzufolge auf dem Wunsch, den Ermessensspielraum der Interviewer im Vergleich zu einer willkürlichen Auswahl durch Konstruktion einer „quotenkontrollierten“ Stichprobe einzuengen. Anläßlich der amerikanischen Präsidentschaftswahlen des Jahres 1936, bei denen sich der Demokrat Franklin Roosevelt und der Republikaner Alfred Landon gegenüberstanden, bestand diese von Gallup, Cherington, Roper und Crossley entwickelte Methode ihre erste große Bewährungsprobe (vgl. etwa Bortz, Döring, 1995, S. 372): Die Zeitschrift „Literary Digest“" verwendete die alten Methoden mit enormen Stichprobenumfängen, die anläßlich der Wahlen zuvor richtige Prognosen des Wahlausganges lieferten. Gallup befragte hingegen lediglich 4000 Personen und prognostizierte im Gegensatz zum „Literary Digest“ einen Wahlsieg Roosevelts. Roosevelt gewann die Wahl mit 62\% der abgegebenen Stimmen. Das Quotenverfahren etablierte sich sofort als die bevorzugte Auswahlmethode der amerikanischen Marktund Meinungsforschung - ein Umstand, an dem auch spätere Mißerfolge, wie anläßlich der Präsidentschaftswahlen von 1948 (vgl. etwa Kruskal, Mosteller, 1980, S. 191) nichts änderte. Es war nicht theoretisch fundiert, hatte aber spektakulären Erfolg. Im Anschluß an diesen Wahlprognoseerfolg erlebten die amerikanischen Markt- und Meinungsforschungsinstitute einen rasanten Aufstieg. Neben den Unternehmern, die sich von den Marktforschern das Verbrauchsverhalten der potentiellen Kunden vorhersagen ließen, bedienten sich sogleich auch Politiker der Institute als Informationsquelle über die „öffentliche Meinung“. So ließ sich etwa Roosevelt den Kriegseintritt genauso durch eine landesweite Gallupumfrage sanktionieren wie später die Bombardierung Roms durch eine Umfrage unter den amerikanischen Katholiken (vgl. Kotz, Johnson, 1986, S. 354). Schon während dieser Kriegsjahre übernahmen neugegründete Institute auch in ganz Europa diese Auswahltechnik.

\subsection{Die Auswahl nach kombinierten Quoten (unter Urnen- modellbedingungen)}

Die Auswahl nach kombinierten Quoten entspricht unter der - in Abschnitt 1 dieser Arbeit motivierten - Annahme gleicher Auswahlchancen für die Untersuchungsobjekte einer geschichteten Zufallsauswahl mit proportionaler Allokation des Stichprobenumfanges auf die Schichten, wobei die Schichtung nach einem m-dimensionalen Merkmal ABC..., erfolgt. Die Verallgemeinerung der Theorie des Schichtenverfahrens auf ein mehrdimensionales Schichtmerkmal eröffnet keinerlei Probleme, so daß die Auswahl nach kombinierten Quoten unter Urnenmodellbedingungen die Eigenschaften des Schichtenverfahrens, wie die Erwartungstreue der Schätzer und den Genauigkeitsgewinn im Vergleich zu einer uneingeschränkten Zufallsauswahl für sich beanspruchen kann (vgl. etwa Stenger, 1986, S. 115ff; Quatember, 1994; Quatember, 1996c). 
Es gilt demnach (um umständliche Notationen zu vermeiden, beschränkt sich die formale Darstellung auf die Verwendung von 2 Quotenmerkmalen A und B mit $r$ bzw. s Ausprägungen):

$$
\overline{\mathrm{x}}_{\mathrm{kQ}}=\sum_{\mathrm{i}=1}^{\mathrm{r}} \sum_{\mathrm{j}=1}^{\mathrm{s}} \pi_{\mathrm{ij}} \cdot \overline{\mathrm{x}}_{\mathrm{ij}}
$$

ist erwartungstreuer Schätzer für den Parameter $\mu$ des Untersuchungsmerkmals $\mathbf{X} . \pi_{\mathrm{ij}}$ ist der Anteil an Objekten mit der Ausprägung $\mathrm{A}_{\mathrm{i}} \mathrm{B}_{\mathrm{j}}$ des Quotenmerkmals $\mathbf{A B}$ in der Grundgesamtheit. $\mathrm{X}_{\mathrm{ij}}$ ist der Stichprobenmittelwert des Untersuchungsmerkmals $\mathbf{X}$ innerhalb der durch die Merkmalsausprägung $\mathrm{A}_{\mathrm{i}} \mathrm{B}_{\mathrm{j}}$ von $\mathbf{A B}$ definierten Teilgesamtheit mit $\mathrm{E} x_{i j}=\mu_{i j}$.

Hinsichtlich der Varianz des Mittelwertschätzers $x_{k Q}$ gilt für Ziehen mit Zurücklegen:

$$
\operatorname{Var} \overline{\mathrm{x}}_{\mathrm{kQ}}=\frac{1}{\mathrm{n}} \sum_{\mathrm{i}=1}^{\mathrm{r}} \sum_{\mathrm{j}=1}^{\mathrm{s}} \pi_{\mathrm{ij}} \cdot \sigma_{\mathrm{ij}}^{2}
$$

mit $\sigma_{\mathrm{ij}}^{\llcorner}$, der Varianz des Merkmals $\mathbf{X}$ innerhalb der Klasse ij des Quotenmerkmals AB in der Grundgesamtheit.

\subsection{Die Auswahl nach Randquoten (unter Urnenmodell- bedingungen)}

Wie aber verhält es sich mit den eben untersuchten statistischen Eigenschaften bei Schätzern, die aus Quotenauswahlen nach Randquoten stammen? Um umständliche Notationen zu vermeiden, beschränkt sich die formale Darstellung der Überprüfung der Erwartungstreue eines Mittelwertschätzers wieder auf die Verwendung zweier Quotenmerkmale A und B mir $r$ bzw. s Ausprägungen. Erhoben wird die Statistik $\bar{x}_{R Q}$ mit

$$
\overline{\mathrm{x}}_{\mathrm{RQ}}=\sum_{\mathrm{i}=1}^{\mathrm{r}} \sum_{\mathrm{j}=1}^{\mathrm{s}} \mathrm{p}_{\mathrm{ij}} \cdot \overline{\mathrm{x}}_{\mathrm{ij}}
$$

worin $\mathrm{p}_{\mathrm{ij}}$ der bei Auswahl nach Randquoten variable Anteil der Stichprobe mit der kombinierten Ausprägung $A_{i} B_{j}$ (also z.B. Frauen einer bestimmten Altersklasse) und $\bar{x}_{i j}$ der Stichprobenmittelwert des Untersuchungsmerkmals $\mathbf{X}$ innerhalb der durch $\mathrm{A}_{\mathrm{i}} \mathrm{B}_{\mathrm{j}}$ definierten Teilgesamtheit ist.

Die Überprüfung der Erwartungstreue dieses Schätzers führt zu folgender Problemstellung:

$$
\begin{aligned}
E \bar{x}_{R Q} & =\sum_{i} \sum_{j} E\left(p_{i j} \cdot \bar{x}_{i j}\right)=\sum_{i} \sum_{j} E\left(E\left(p_{i j} \cdot \bar{x}_{i j} \mid p_{i j}\right)\right)= \\
& =\sum_{i} \sum_{j} E\left(p_{i j} \cdot E\left(\bar{x}_{i j} \mid p_{i j}\right)\right)
\end{aligned}
$$


(vgl. Quatember, 1996b, S. 65). Abgesehen vom trivialen Fall, daß alle $E\left(\bar{x}_{i j} \mid p_{i j}\right)=\mu$ sind, ist $\overline{\mathrm{x}}_{\mathrm{RQ}}$ somit erwartungstreuer Schätzer für $\mu$ dann und nur dann, wenn

$$
E\left(\bar{x}_{i j} \mid p_{i j}\right)=\mu_{i j}
$$

und

$$
\mathrm{E} \mathrm{p}_{\mathrm{ij}}=\pi_{\mathrm{ij}} \text {, }
$$

beide Zufallsvariable also erwartungstreue Schätzer für die jeweiligen Parameter sind. Forderung (4) hält, da innerhalb jeder Teilgesamtheit per definitionem eine uneingeschränkte Zufallsauswahl der Objekte vorgenommen wird. Eine Besonderheit des Auswahlvorganges bei Auswahl nach Randquoten unter Urnenmodellbedingungen, nämlich die Beendigung der uneingeschränkten Suche nach Untersuchungsobjekten mit Erfüllung der ersten vorgegebenen Randquote (z.B. wenn schon eine genügend große Anzahl an Männern befragt wurde), steht jedoch der formalen Überprüfung von Forderung (5) im Wege.

Als Ausweg bleibt die exakte numerische Berechnung des Erwartungswertes in Beispielen und die auf diesen Ergebnissen beruhende Überprüfung des Haltens von (5). Folgendes Beispiel widmet sich dieser Aufgabenstellung:

Beispiel 1: Gegeben sei folgende Verteilung der Grundgesamtheit auf den eindimensionalen Quotenmerkmalen A und B:

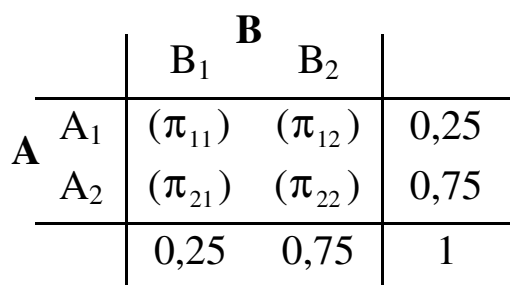

Die Randverteilungen sind vorgegeben, die zweidimensionale Verteilung der Grundgesamtheit auf dem Merkmal AB ist unbekannt. Eine Auswahl nach Randquoten vom Umfang $\mathrm{n}=4$ aus dieser Grundgesamtheit kann nur eine der beiden folgenden Kontingenztafeln $\mathrm{T}_{1}$ und $\mathrm{T}_{2}$ erzeugen:

\begin{tabular}{l|cc|c}
$\mathrm{T}_{1}:$ & $\mathrm{B}_{1}$ & $\mathrm{~B}_{2}$ & \\
\hline $\mathrm{A}_{1}$ & 1 & 0 & 1 \\
$\mathrm{~A}_{2}$ & 0 & 3 & 3 \\
\hline & 1 & 3 & 4
\end{tabular}

$\mathrm{T}_{2}:$\begin{tabular}{c|cc|c} 
& $\mathrm{B}_{1}$ & $\mathrm{~B}_{2}$ & \\
\hline $\mathrm{A}_{1}$ & 0 & 1 & 1 \\
$\mathrm{~A}_{2}$ & 1 & 2 & 3 \\
\hline & 1 & 3 & 4
\end{tabular}

Im Vergleich dazu wären bei uneingeschränkter Zufallsauswahl 35 verschiedene Aufteilungen der 4 Objekte auf die 4 Felder möglich. Die Bestimmung jener Abläufe der Stichprobenauswahl, die zur Kontingenztafel $T_{v}$ führen und die Vorgabe der zweidimensionalen Parameter $\pi_{\mathrm{ij}}$ ermöglichen die Berechnung der Eintreffwahrscheinlichkeiten für diese Kontingenztafeln und erlauben somit die Berechnung von

$$
E p_{i j}=\sum_{v=1}^{t} p_{i j \mid T_{v}} \cdot \operatorname{Pr}\left(T_{v}\right)
$$


(t... Anzahl der verschiedenen möglichen Kontingenztafeln). In Beispiel 1 mit $\mathrm{t}=2$ gibt es 4 verschiedene Abläufe der Stichprobenauswahl, die zur Erzeugung der Tafel $\mathrm{T}_{1}$ führen (vgl. ebd., S. 67): Wird beispielsweise beim 1. Zug ein Objekt ausgewählt, das die Ausprägung $A_{1} B_{1}$ aufweist, so sind die Randquoten bezüglich der Ausprägungen $A_{1}$ und $\mathrm{B}_{1}$ bereits erfüllt und es bleibt (dem Interviewer) nur mehr die Aufgabe, 3 Objekte $\mathrm{zu}$ finden, die allesamt die Ausprägung $\mathrm{A}_{2} \mathrm{~B}_{2}$ aufweisen. Die Kontingenztafel $\mathrm{T}_{1}$ wäre damit bereits mit dem 1. Ziehen eines Objektes für die Stichprobe realisiert und dieser Ablauf der Stichprobenziehung tritt mit Wahrscheinlichkeit $\pi_{11}$ ein. Tabelle 1 beinhaltet auch die 3 weiteren Abläufe, welche $\mathrm{T}_{1}$ realisieren.

Tabelle 1: Abläufe der Stichprobenverteilung, die in Beispiel 1 zur Tafel $T_{1}$ führen.

\begin{tabular}{|c|c|c|c|c|}
\hline & \multicolumn{4}{|c|}{ Stichprobenauswahl } \\
Ablauf & 1. Objekt & 2. Objekt & 3. Objekt & 4. Objekt \\
\hline 1 & $\mathrm{~A}_{1} \mathrm{~B}_{1}$ & $\left(\mathrm{~A}_{2} \mathrm{~B}_{2}\right)$ & $\left(\mathrm{A}_{2} \mathrm{~B}_{2}\right)$ & $\left(\mathrm{A}_{2} \mathrm{~B}_{2}\right)$ \\
2 & $\mathrm{~A}_{2} \mathrm{~B}_{2}$ & $\mathrm{~A}_{1} \mathrm{~B}_{1}$ & $\left(\mathrm{~A}_{2} \mathrm{~B}_{2}\right)$ & $\left(\mathrm{A}_{2} \mathrm{~B}_{2}\right)$ \\
3 & $\mathrm{~A}_{2} \mathrm{~B}_{2}$ & $\mathrm{~A}_{2} \mathrm{~B}_{2}$ & $\mathrm{~A}_{1} \mathrm{~B}_{1}$ & $\left(\mathrm{~A}_{2} \mathrm{~B}_{2}\right)$ \\
4 & $\mathrm{~A}_{2} \mathrm{~B}_{2}$ & $\mathrm{~A}_{2} \mathrm{~B}_{2}$ & $\mathrm{~A}_{2} \mathrm{~B}_{2}$ & $\mathrm{~A}_{1} \mathrm{~B}_{1}$ \\
\hline
\end{tabular}

Durch Vorgabe der Parameter $\pi_{\mathrm{ij}}$ lassen sich nun die Erwartungswerte von $\mathrm{p}_{\mathrm{ij}}$ (für Ziehen mit Zurücklegen) berechnen. Diese sind in Abbildung 1 für das Element $\mathrm{p}_{11}$ über alle in Beispiel 1 möglichen Werte für $\pi_{11}$ nach oben aufgetragen:

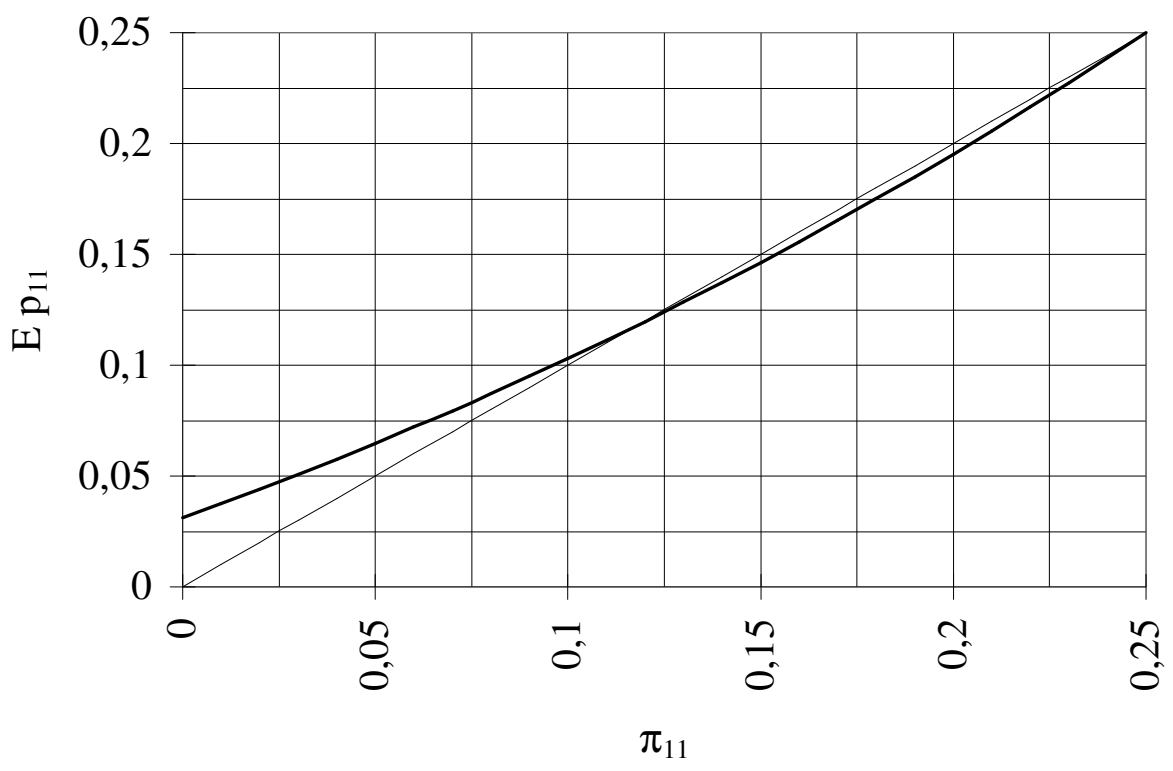

Abbildung 1: Abweichungen von $\mathrm{E} \mathrm{p}_{11}$ vom Parameter $\pi_{11}$ in Abhängigkeit von $\pi_{11}$ bei allen in Beispiel 1 möglichen Werten für $\pi_{11}$.

Bei den zweidimensionalen Parametern $\pi_{11}=0,05, \pi_{12}=\pi_{21}=0,20$ und $\pi_{22}=0,55$ gilt z.B.: $\mathrm{E}_{11}=0,06475$, wie mit Hilfe von Tabelle 1 für Ziehen mit Zurücklegen leicht nachzuvollziehen ist: 


$$
\operatorname{Pr}\left(\mathrm{T}_{1}\right)=0,05+0,05 \cdot 0,55+0,05 \cdot 0,55^{2}+0,55^{3}=0,259
$$

und somit

$$
\operatorname{Pr}\left(\mathrm{T}_{2}\right)=1-0,259=0,741 .
$$

(6) ergibt dann:

$$
\mathrm{E}_{11}=0,25 \cdot 0,259+0 \cdot 0,741=0,06475 \text {. }
$$

Die Behauptung, daß für Auswahlen nach Randquoten Forderung (5) hält, ist wegen

$$
\mathrm{E}_{11}=0,06475 \neq 0,05=\pi_{11}
$$

somit bereits falsifiziert (vgl. Abbildung 1 für alle möglichen Werte von $\pi_{11}$ ). Weiters gilt demnach:

$$
\mathrm{E}_{12}=\mathrm{E} \mathrm{p}_{21}=0,18525 \neq 0,20=\pi_{12}=\pi_{21}
$$

und

$$
\mathrm{E}_{22}=0,56475 \neq 0,55=\pi_{22} \text {. }
$$

Es gibt tatsächlich in Abbildung 1 nur einen einzigen nicht näher definierbaren Punkt, der etwa bei $\pi_{11}^{\prime}=0,118$ liegt, bei dem $E \mathrm{p}_{11}=\pi_{11}$, da an dieser Stelle die durchschnittliche Überschätzung des Parameters $\pi_{11}$ durch $p_{11}$ für $\pi_{11} \in\left[0 ; \pi_{11}^{\prime}\right\rfloor$ in eine Unterschätzung von $\pi_{11}$ für $\pi_{11} \in\left[\pi_{11}^{\prime} ; 0,25\right]$ übergeht.

Betrachten wir folgendes Beispiel:

Beispiel 2: Gegeben sei folgende Verteilung der Grundgesamtheit auf den eindimensionalen Quotenmerkmalen A und B:

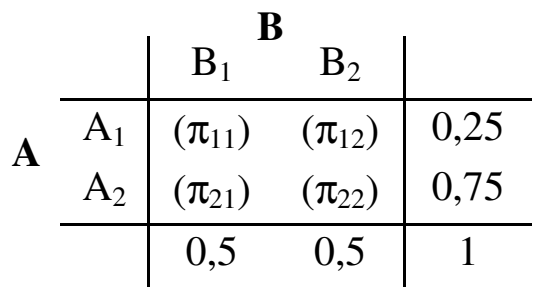

Eine Auswahl nach Randquoten vom Umfang $\mathrm{n}=4$ aus dieser Grundgesamtheit kann nur eine der beiden folgenden Kontingenztafeln $\mathrm{T}_{1}$ und $\mathrm{T}_{2}$ erzeugen:

\begin{tabular}{l|cc|c}
$\mathrm{T}_{1}:$ & $\mathrm{B}_{1}$ & $\mathrm{~B}_{2}$ & \\
\hline $\mathrm{A}_{1}$ & 1 & 0 & 1 \\
$\mathrm{~A}_{2}$ & 1 & 2 & 3 \\
\hline & 2 & 2 & 4
\end{tabular}

T2: \begin{tabular}{l|cc|c} 
& $\mathrm{B}_{1}$ & $\mathrm{~B}_{2}$ & \\
\hline $\mathrm{A}_{1}$ & 0 & 1 & 1 \\
$\mathrm{~A}_{2}$ & 2 & 1 & 3 \\
\hline & 2 & 2 & 4
\end{tabular}

Wie leicht nachvollziehbar ist, gibt es nun 8 verschiedene Abläufe der Stichprobenauswahl, die zur Realisierung der Kontingenztafel $T_{1}$ führen. Wieder ist die Tafel bereits nach dem 1. Zug realisiert worden, wenn als 1. Element ein Objekt mit Ausprägung $A_{1} B_{1}$ gezogen wurde. Es sind dann noch ein Element mit $A_{2} B_{1}$ und zwei Elemente mit $\mathrm{A}_{2} \mathrm{~B}_{2}$ zu suchen, wobei die Reihenfolge des Auffindens dieser Objekte für die Eintreffwahrscheinlichkeit der Kontingenztafel $\mathrm{T}_{1}$ ohne Belang ist. Die Wahrscheinlichkeit dieses Ablaufes beträgt daher abermals $\pi_{11}$. Durch Berücksichtigung aller 
möglichen Abläufe der Stichprobenauswahl und Vorgabe aller möglichen Parameter lassen sich mit Hilfe der Eintreffwahrscheinlichkeit für die Kontingenztafel $\mathrm{T}_{1}$ wieder die Erwartungswerte von $p_{i j}$ in Abhängigkeit von $\pi_{\mathrm{ij}}$ bestimmen. Diese sind für das Element $\mathrm{p}_{11}$ in Abbildung 2 dargestellt:

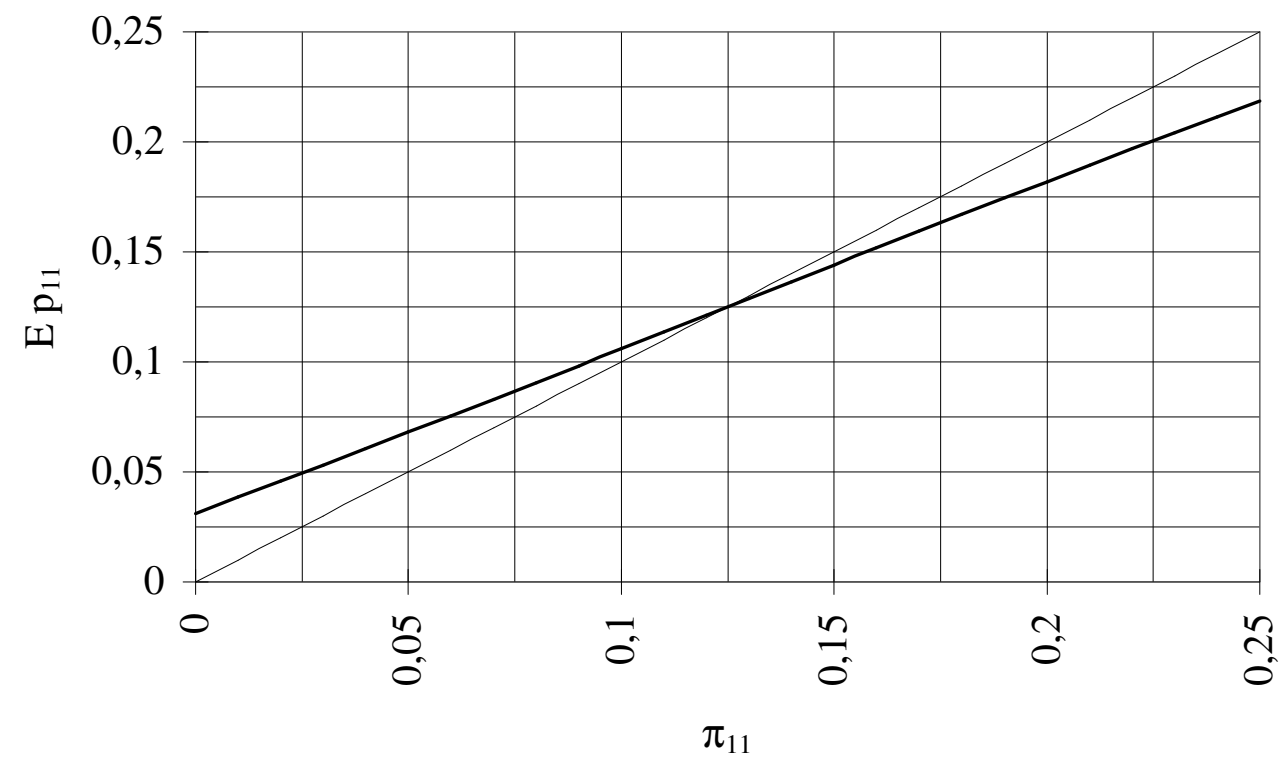

Abbildung 2: Abweichungen von $\mathrm{E} \mathrm{p}_{11}$ vom Parameter $\pi_{11}$ in Abhängigkeit von $\pi_{11}$ bei allen in Beispiel 2 möglichen Werten für $\pi_{11}$.

Aus Abbildung 2 geht hervor, daß es bei den in Beispiel 2 vorgegebenen Randquoten wiederum nur einen einzigen Wert von $\pi_{11}$ gibt, für den Forderung (5) gilt. Es ist dies exakt $\pi_{11}^{\prime}=0,125$.

Betrachten wir noch ein weiteres Beispiel:

Beispiel 3: Gegeben sei folgende Verteilung der Grundgesamtheit auf den eindimensionalen Quotenmerkmalen A und B:

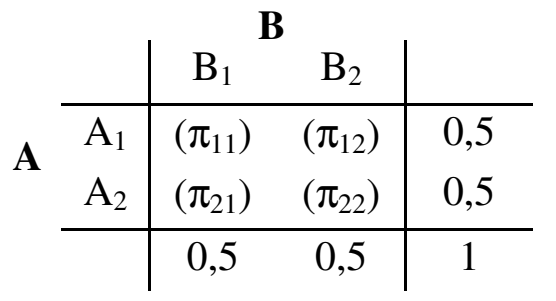

Eine Auswahl nach Randquoten vom Umfang $n=4$ aus dieser Grundgesamtheit kann nun eine der drei folgenden Kontingenztafeln erzeugen:

\begin{tabular}{l|cc|c}
$\mathrm{T}_{1}:$ & $\mathrm{B}_{1}$ & $\mathrm{~B}_{2}$ & \\
\hline $\mathrm{A}_{1}$ & 2 & 0 & 2 \\
$\mathrm{~A}_{2}$ & 0 & 2 & 2 \\
\hline & 2 & 2 & 4
\end{tabular}

\begin{tabular}{l|cc|c}
$\mathrm{T}_{2}:$ & $\mathrm{B}_{1}$ & $\mathrm{~B}_{2}$ & \\
\hline $\mathrm{A}_{1}$ & 1 & 1 & 2 \\
$\mathrm{~A}_{2}$ & 1 & 1 & 2 \\
\hline & 2 & 2 & 4
\end{tabular}

$\mathrm{T}_{3}:$\begin{tabular}{c|cc|c} 
& $\mathrm{B}_{1}$ & $\mathrm{~B}_{2}$ & \\
\hline $\mathrm{A}_{1}$ & 0 & 2 & 2 \\
$\mathrm{~A}_{2}$ & 2 & 0 & 2 \\
\hline & 2 & 2 & 4
\end{tabular}


Die exakte numerische Berechnung der Erwartungswerte für $\mathrm{p}_{\mathrm{ij}}$ wie in den Beispielen 1 und 2 führt bei variablem Parameter $\pi_{\mathrm{ij}}$ jetzt zu folgender Darstellung der Abhängigkeit von $\mathrm{E}_{11}$ von $\pi_{11}$ :

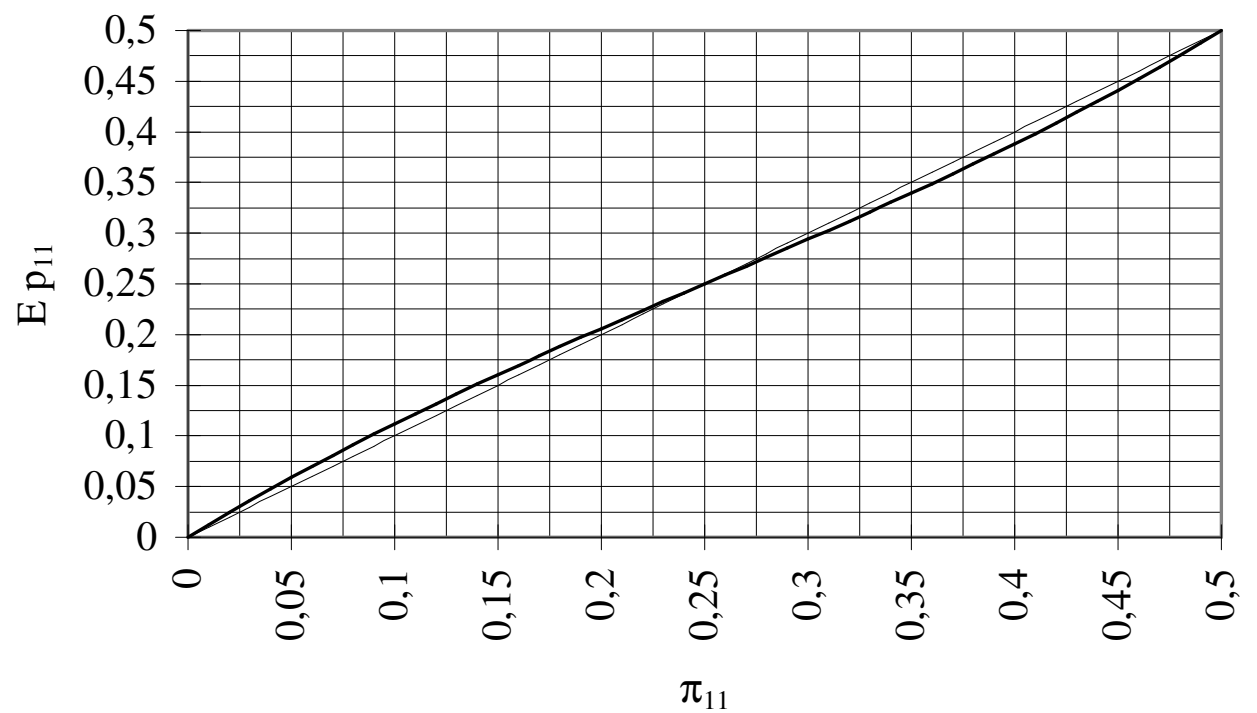

Abbildung 3: Abweichungen von $\mathrm{E} \mathrm{p}_{11}$ vom Parameter $\pi_{11}$ in Abhängigkeit von $\pi_{11}$ bei allen in Beispiel 3 möglichen Werten für $\pi_{11}$.

Wiederum schneidet die Kurve der Erwartungswerte der kombinierten Quoten in der Stichprobe $\mathrm{p}_{11}$ die Gerade erwartungstreuer Schätzer für $\pi_{11}$ lediglich in einem Punkt aus dem für $\pi_{11}$ möglichen Wertebereich. Dies ist diesmal der Punkt bei $\pi_{11}^{\prime}=0,25$.

Die Schnittpunkte der E $\mathrm{p}_{11}$-Kurven und der $\pi_{11}$-Geraden liegen in den Beispielen 2 und 3 - im Gegensatz zum Beispiel 1 - offensichtlich bei einem genau definierten Punkt: die Kurven schneiden sich bei $\pi_{11}=\pi_{1} \cdot \pi_{.1}$. Die Zellenparameter werden in diesen Beispielen also erwartungstreu geschätzt, wenn die Quotenmerkmale A und B voneinander statistisch unabhängig sind.

Die Betrachtung der Ergebnisse solcher Überprüfungen für alle möglichen Vierfeldertafeln mit Stichprobenumfängen zwischen 3 und 10 bei $\pi_{\mathrm{ij}}=\pi_{\mathrm{i} .} \cdot \pi_{\mathrm{j}}$ offenbart (vgl. ebd., S. 101ff), daß tatsächlich bei Unabhängigkeit der beiden Quotenmerkmale A und $\mathbf{B}$ und gleichzeitiger Gleichverteilung mindestens eines der beiden Merkmale eine erwartungstreue Schätzung der (kombinierten) Zellenparameter $\pi_{\mathrm{ij}}$ erfolgt. Dies ist auch begründbar: In Beispiel 2 etwa unterscheiden sich die beiden möglichen Kontingenztafeln nur durch die Vertauschung der Spalten. Dadurch sind die Abläufe des Ziehens, die zur Erzeugung dieser Tafeln führen, natürlich exakt spiegelbildlich. Wegen der Unabhängigkeit von A und $\mathbf{B}$ sind die zweidimensionalen Zellenparameter $\pi_{\mathrm{ij}}$ zeilenweise identisch, denn das Merkmal B ist gleichverteilt. Da die (vertauschten) Spalten der spiegelbildlichen Abläufe daher wieder auf dieselben Zellenparameter treffen, sind die Wahrscheinlichkeiten für das Eintreffen dieser beiden Tafeln ident. Die Symmetrie in den Wahrscheinlichkeiten aller möglichen Kontingenztafeln bei Unabhängigkeit der Quotenmerkmale und Gleichverteilung eines Merkmals bewirkt nun aber, daß die Erwartungswerte der relativen Zellenhäufigkeiten der Stichprobe $E p_{i j}$ zeilenweise 
gleich und wegen des Einhaltens der vorgegebenen Randquoten daher mit den Parametern $\pi_{\mathrm{ij}}$ identisch sein müssen. Vollkommen analoge Überlegungen gelten für 2 gleichverteilte Quotenmerkmale, wobei die Symmetrie dann sowohl in den Spalten als auch in den Zeilen existiert (vgl. ebd., S. 69f).

Die Verallgemeinerung dieser Aussagen auf allgemeine $\mathrm{r} \times \mathrm{s}-$ Tafeln birgt ausschließlich das Problem komplexerer Abläufe der Objekteauswahl aufgrund höherer Freiheitsgrade.

Beispiel 4: Gegeben sei folgende Verteilung der Grundgesamtheit auf den eindimensionalen Quotenmerkmalen A und B:

\begin{tabular}{|c|c|c|c|c|c|}
\hline & \multicolumn{4}{|c|}{ B } \\
\hline & & $\mathrm{B}_{1}$ & $\mathrm{~B}_{2}$ & $\mathrm{~B}_{3}$ & \\
\hline \multirow{4}{*}{ A } & $\mathrm{A}_{1}$ & $\left(\pi_{11}\right)$ & $\left(\pi_{12}\right)$ & $\ldots$ & $1 / 3$ \\
\hline & $\mathrm{A}_{2}$ & $\left(\pi_{21}\right)$ & & & $1 / 3$ \\
\hline & $\mathrm{A}_{3}$ & & & & $1 / 3$ \\
\hline & & $1 / 3$ & $1 / 3$ & $1 / 3$ & 1 \\
\hline
\end{tabular}

Eine Auswahl nach Randquoten vom Umfang $n=3$ aus dieser Grundgesamtheit kann eine von 6 möglichen Kontingenztafeln realisieren. Die exakte numerische Berechnung der Erwartungswerte der relativen Zellenhäufigkeiten E $p_{i j}$ weist nun bei allen $\pi_{\mathrm{ij}}$ Verzerrungen nach - mit Ausnahme von $\pi_{\mathrm{ij}}=\pi_{\mathrm{i} .} \cdot \pi_{\mathrm{j}}=1 / 9$, also der bei Unabhängigkeit der Merkmale A und $\mathbf{B}$ auftretenden Zellenparameter. Die Betrachtung der 6 möglichen Kontingenztafeln macht in Beispiel 4 augenscheinlich, daß auch hier die Spiegelbildlichkeit der Abläufe der Stichprobenauswahl als Begründung für dieses Faktum ausreicht.

Bewirkt die Hinzunahme eines weiteren Quotenmerkmals eine Veränderung hinsichtlich der Überprüfung der Erwartungstreue der Schätzer für die kombinierten Quoten? Betrachten wir zur Beantwortung dieser Frage nochmals Beispiel 1:

Beispiel 5: Gegeben sei folgende Verteilung der Grundgesamtheit auf dem Quotenmerkmal $\mathbf{C}$ mit Ausprägungen $\mathrm{C}_{1}$ und $\mathrm{C}_{2}$ (die Verteilung der Grundgesamtheit auf den Merkmalen A und $\mathbf{B}$ entspricht jener aus Beispiel 1):
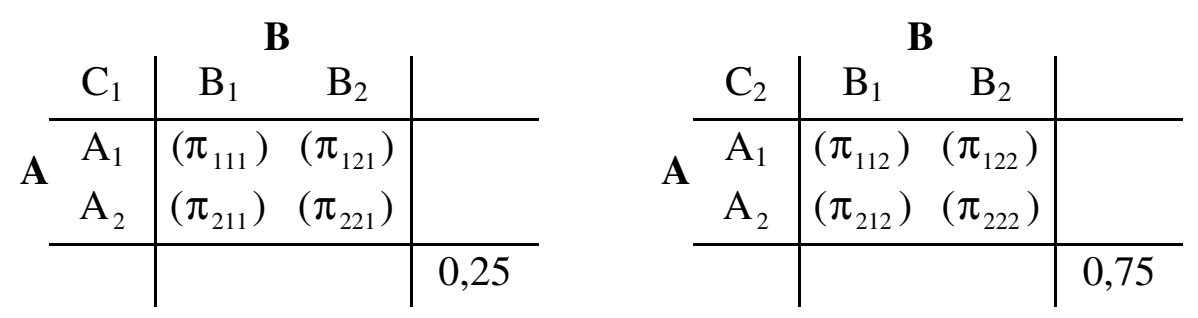

Eine Auswahl nach Randquoten vom Umfang $n=4$ führt unter Urnenmodellbedingungen $\mathrm{zu}$ einer von 5 möglichen Verteilungen der Stichprobe auf dem dreidimensionalen Merkmal ABC. Wird etwa beispielsweise als erstes zufällig ein Objekt mit Ausprägung $\mathrm{A}_{1} \mathrm{~B}_{1} \mathrm{C}_{1}$ aus der Grundgesamtheit gezogen, so ist, da damit bereits die vorgegebenen Randquoten hinsichtlich der Ausprägungen $A_{1}, B_{1}$ und $C_{1}$ erfüllt, sind folgende Stichprobenverteilung auf dem Merkmal ABC realisiert: 


\begin{tabular}{c|cc|c}
$\mathrm{C}_{1}$ & $\mathrm{~B}_{1}$ & $\mathrm{~B}_{2}$ & \\
\hline $\mathrm{A}_{1}$ & 1 & 0 & \\
$\mathrm{~A}_{2}$ & 0 & 0 & \\
\hline & & & 1
\end{tabular}

\begin{tabular}{c|cc|c}
$\mathrm{C}_{2}$ & $\mathrm{~B}_{1}$ & $\mathrm{~B}_{2}$ & \\
\hline $\mathrm{A}_{1}$ & 0 & 0 & \\
$\mathrm{~A}_{2}$ & 0 & 3 & \\
\hline & & & 3
\end{tabular}

Es gilt dann nach Ziehung des ersten Elementes (für den Interviewer) nur mehr, 3 Objekte ausfindig zu machen, welche die Ausprägung $\mathrm{A}_{2} \mathrm{~B}_{2} \mathrm{C}_{2}$ aufweisen.

Durch die Bestimmung aller möglichen Abläufe der Stichprobenauswahl können wieder die Eintreffwahrscheinlichkeiten für die 5 verschiedenen möglichen Stichprobenverteilungen auf dem Merkmal ABC berechnet werden, wobei durch die Hinzunahme eines dritten Merkmals genauso wie durch Erhöhung der Ausprägungen pro Merkmal (siehe Beispiel 4) nach Erfüllung nur einer Randquote die Verteilung der restlichen Objekte auf dem Quotenmerkmal ABC noch nicht vollständig bestimmt ist, aber die Möglichkeiten dafür eingeschränkt sind, und daher ab diesem Zeitpunkt bedingte Wahrscheinlichkeiten bei der Berechnung dieser Eintreffwahrscheinlichkeiten entstehen. Überprüft man mit diesen Wahrscheinlichkeiten $\operatorname{Pr}\left(T_{v}\right)$ wiederum die Erwartungstreue des Schätzers für die kombinierten Quoten $\mathrm{p}_{\mathrm{ijk}}$ durch die Berechnung von

$$
E \mathrm{p}_{\mathrm{ijk}}=\sum_{\mathrm{v}=1}^{\mathrm{t}} \mathrm{p}_{\mathrm{ijk} \mid \mathrm{T}_{\mathrm{v}}} \cdot \operatorname{Pr}\left(\mathrm{T}_{\mathrm{v}}\right)
$$

( $\mathrm{t}$... Anzahl der verschiedenen möglichen Verteilungen der Stichprobe auf dem Merkmal ABC), so stellt sich das nach den Ergebnissen von Beispiel 1 zu erwartende Ergebnis ein: die Hypothese $E \mathrm{p}_{\mathrm{ijk}}=\pi_{\mathrm{ijk}}$ wird falsifiziert. Bei den dreidimensionalen Parametern $\pi_{111}=\pi_{121}=\pi_{211}=\pi_{112}=0,05, \pi_{221}=\pi_{122}=\pi_{212}=0,10$ und $\pi_{222}=0,50$ gilt z.B. (an der 4. Nachkommastelle gerundet): $\mathrm{E}_{111}=0,0531 \neq 0,05=\pi_{111}$ usf. Auch die Unabhängigkeit der 3 Quotenmerkmale untereinander läßt wie in Beispiel 1 keine erwartungstreue Schätzung der kombinierten Quoten zu, denn hinsichtlich der relativen Häufigkeit $\mathrm{p}_{111}$ ergibt sich in der Auswahl nach Randquoten vom Umfang $\mathrm{n}=4$ in diesem Fall:

$$
\mathrm{E}_{111}=0,0250 \neq 0,0156=\pi_{111}
$$

Erst wenn zumindest zwei der drei Randverteilungen Gleichverteilungen sind, gilt bei Unabhängigkeit der Quotenmerkmale, also wenn $\pi_{\mathrm{ijk}}=\pi_{\mathrm{i..}} \cdot \pi_{. \mathrm{j} .} \cdot \pi_{. . \mathrm{k}}(\forall \mathrm{i}, \mathrm{j}, \mathrm{k})$ : E $\mathrm{p}_{\mathrm{ijk}}=$ $\pi_{\mathrm{ijk}}$. Dieser Umstand kann durch die Ergebnisse der Betrachtung zweidimensionaler Quotenmerkmale (Beispiele 2 und 3) erklärt werden: Die dort als Begründung für die punktuelle Erwartungstreue bei Unabhängigkeit der Quotenmerkmale angeführte Spiegelbildlichkeit der Stichprobenabläufe und Symmetrie in den Eintreffwahrscheinlichkeiten der verschiedenen möglichen Kontingenztafeln ist bei Verwendung von drei Quotenmerkmalen eben erst bei 2 Gleichverteilungen unter den 3 eindimensionalen Randverteilungen gegeben (Beispiel 6). 
Beispiel 6: Gegeben sei folgende Verteilung der Grundgesamtheit auf dem Quotenmerkmal $\mathbf{C}$ mit Ausprägungen $\mathrm{C}_{1}$ und $\mathrm{C}_{2}$ (die Verteilung der Grundgesamtheit auf den Merkmalen A und B entspricht jener aus Beispiel 3):

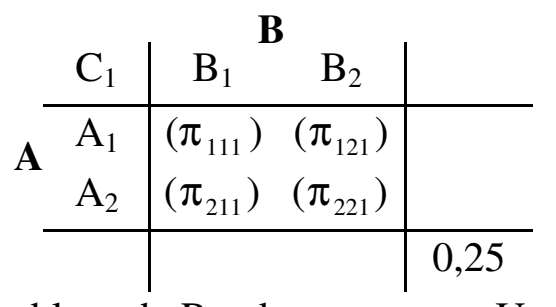

A \begin{tabular}{c|cc|c}
$\mathrm{C}_{2}$ & $\mathrm{~B}_{1}$ & $\mathrm{~B}_{2}$ & \\
\hline $\mathrm{A}_{1}$ & $\left(\pi_{112}\right)$ & $\left(\pi_{122}\right)$ & \\
$\mathrm{A}_{2}$ & $\left(\pi_{212}\right)$ & $\left(\pi_{222}\right)$ & \\
\hline & & & 0,75
\end{tabular}

Eine Auswahl nach Randquoten vom Umfang $\mathrm{n}=4$ führt $\mathrm{zu}$ folgenden möglichen Verteilungen der Stichprobe auf dem Merkmal ABC:

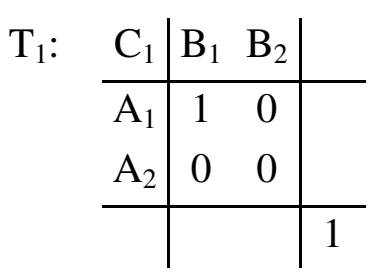

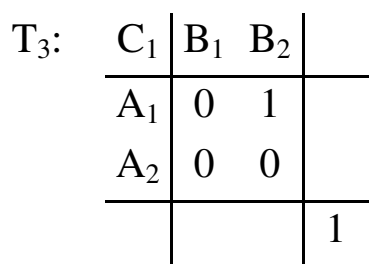

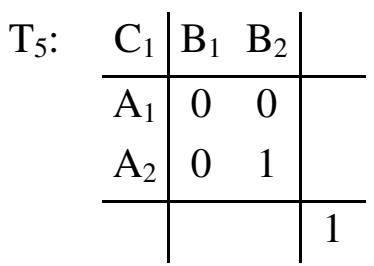

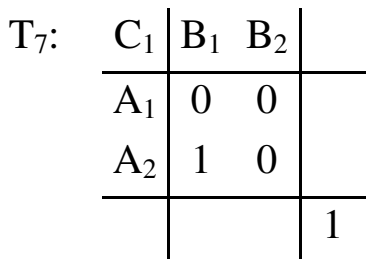

\begin{tabular}{c|cc|c}
$\mathrm{C}_{2}$ & $\mathrm{~B}_{1}$ & $\mathrm{~B}_{2}$ & \\
\hline $\mathrm{A}_{1}$ & 1 & 0 & \\
$\mathrm{~A}_{2}$ & 0 & 2 & \\
\hline & & 3
\end{tabular}

\begin{tabular}{c|cc|c}
$\mathrm{C}_{2}$ & $\mathrm{~B}_{1}$ & $\mathrm{~B}_{2}$ & \\
\hline $\mathrm{A}_{1}$ & 0 & 1 & \\
$\mathrm{~A}_{2}$ & 2 & 0 & \\
\hline & & 3
\end{tabular}

\begin{tabular}{c|cc|c}
$\mathrm{C}_{2}$ & $\mathrm{~B}_{1}$ & $\mathrm{~B}_{2}$ & \\
\hline $\mathrm{A}_{1}$ & 1 & 1 & \\
$\mathrm{~A}_{2}$ & 1 & 0 & \\
\hline & & 3
\end{tabular}

\begin{tabular}{c|cc|c}
$\mathrm{C}_{2}$ & $\mathrm{~B}_{1}$ & $\mathrm{~B}_{2}$ & \\
\hline $\mathrm{A}_{1}$ & 1 & 1 & \\
$\mathrm{~A}_{2}$ & 0 & 1 & \\
\hline & & 3
\end{tabular}
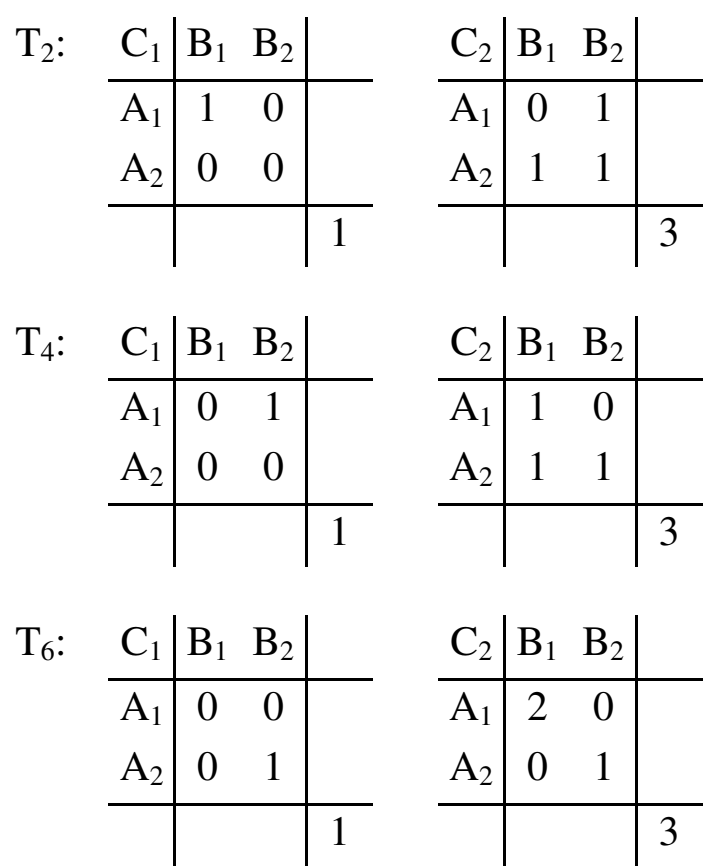
$\mathrm{T}_{8}:$

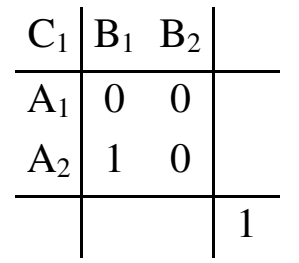

Paarweise spiegelbildliche Abläufe der Stichprobenauswahl (im Sinne der Beispiele 2 und 3) führen zu jenen Stichprobenverteilungen, die in den Tafeln $T_{1}$ und $T_{8}, T_{2}$ und $\mathrm{T}_{7}, \mathrm{~T}_{3}$ und $\mathrm{T}_{6}$ bzw. $\mathrm{T}_{4}$ und $\mathrm{T}_{5}$ (bzw. in den Tafeln $\mathrm{T}_{1}$ und $\mathrm{T}_{3}, \mathrm{~T}_{2}$ und $\mathrm{T}_{4}, \mathrm{~T}_{5}$ und $\mathrm{T}_{7}$ sowie $\mathrm{T}_{6}$ und $\mathrm{T}_{8}$ ) dargestellt sind. Sie unterscheiden sich hier lediglich durch die Vertauschung der Zeilen (bzw. Spalten) in den jeweiligen Tabellen. Bei Unabhängigkeit der Quotenmerkmale A, B und $\mathbf{C}$ treffen die Zeilen (bzw. die Spalten) der spiegelbildlichen Abläufe wiederum auf dieselben Zellenparameter, wodurch diese Tafeln paarweise gleiche Eintreffwahrscheinlichkeiten (von 0,09375 für $\mathrm{T}_{1}$ und $\mathrm{T}_{8}, 0,15626$ für $\mathrm{T}_{2}$ und $\mathrm{T}_{7}$, 0,09375 für $\mathrm{T}_{3}$ und $\mathrm{T}_{6}$ und von 0,15626 für $\mathrm{T}_{4}$ und $\mathrm{T}_{5}$ ) aufweisen. Die Symmetrie in den Wahrscheinlichkeiten für alle möglichen Verteilungen bewirkt nun wieder, daß die Erwartungswerte der relativen Zellenhäufigkeiten der Stichprobe $E p_{i j k}$ pro Tabelle 
identisch sein müssen. Wegen der Vorgabe der einzuhaltenden Randquoten gilt deshalb: $\mathrm{E} \mathrm{p}_{\mathrm{ijk}}=\pi_{\mathrm{ijk}}$. Mindestens 2 Quotenmerkmale müssen hier gleichverteilt sein, um diese Bedingungen herzustellen.

Die Ergebnisse der Betrachtungen zur Erwartungstreue von $\overline{\mathrm{x}}_{\mathrm{RQ}}$ (3) lassen sich nun folgendermaßen zusammenfassen: Eine Quotenauswahl nach Randquoten erzeugt Stichproben, die hinsichtlich der $\mathrm{m}$ eindimensionalen Quotenmerkmale A, B, C, ... ,exakt repräsentativ“ (Quatember, 1996a, S. 237) sind. Wenn der zur oben beschriebenen Herstellung der Symmetrie der möglichen Kontingenztafeln notwendige Teil dieser $\mathrm{m}$ Randverteilungen Gleichverteilungen aufweist, dann sind diese Stichproben unter Urnenmodellbedingungen hinsichtlich des m-dimensionalen Quotenmerkmals ABC... „repräsentativ nach Erwartungswert“ (ebd.), wenn die Quotenmerkmale statistisch unabhängig voneinander sind. Sind weniger als dieser dafür notwendige Teil der $\mathrm{m}$ Randverteilungen Gleichverteilungen, so gibt es zwar ebenfalls Parameterwerte der mdimensionalen Verteilung der Grundgesamtheit auf dem Quotenmerkmal ABC..., die eine erwartungstreue Schätzung dieser kombinierten Quoten zulassen können, diese sind aber nicht die bei statistischer Unabhängigkeit der Quotenmerkmale auftretenden (siehe Abbildung 1).

Im allgemeinen gilt jedoch:

$$
E p_{i j} \neq \pi_{i j}
$$

und daraus folgt:

$$
E \bar{x}_{R Q}=\sum_{i=1}^{r} \sum_{j=1}^{s} \mu_{i j} \cdot E p_{i j} \neq \mu,
$$

sobald nicht alle Schichtmittelwerte $\mu_{\mathrm{ij}}$ übereinstimmen. Der aus einer Quotenstichprobe nach Randquoten erhobene Schätzer $\overline{\mathrm{x}}_{\mathrm{RQ}}$ ist sodann selbst unter Urnenmodellbedingungen nicht erwartungstreu für den Parameter $\mu$ des Untersuchungsmerkmals $\mathbf{X}$.

Erfüllt nun nicht jede Teilstichprobe die vorgegeben Randquoten, sondern nur deren Summe, so läßt sich anhand eines beliebigen Beispiels die Ungültigkeit der Behauptung $\mathrm{E} \mathrm{p}_{\mathrm{ijk}}=\pi_{\mathrm{ijk}}$ sogleich nachweisen:

Beispiel 7: Gegeben sei folgende Verteilung der Grundgesamtheit auf den eindimensionalen Quotenmerkmalen A und B:

\begin{tabular}{cc|cc|c} 
& & \multicolumn{2}{|c}{ B } & \\
& & $\mathrm{B}_{1}$ & $\mathrm{~B}_{2}$ & \\
\hline $\mathrm{A}$ & $\mathrm{A}_{1}$ & $(0,05)$ & $(0,20)$ & 0,25 \\
& $\mathrm{~A}_{2}$ & $(0,20)$ & $(0,55)$ & 0,75 \\
\hline & & 0,25 & 0,75 & 1
\end{tabular}

Eine Auswahl nach Randquoten vom Umfang $\mathrm{n}=8$ wird folgendermaßen auf 2 Interviewer $\left(I_{1}\right.$ und $\left.I_{2}\right)$ aufgeteilt: 

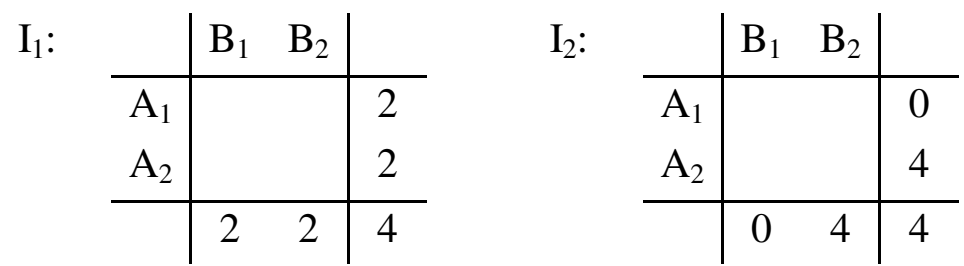

Interviewer $\mathrm{I}_{1}$ hat also seine Teilstichprobe nach nicht proportional zur Grundgesamtheit vorgegebenen Randquoten vom Umfang $n=4$ zu ziehen, während dem Interviewer $\mathrm{I}_{2}$ zur Einhaltung der Randquoten in der Gesamtstichprobe aufgetragen wird, 4 Objekte mit Ausprägung $\mathrm{A}_{2} \mathrm{~B}_{2}$ in seiner Teilstichprobe zu erheben.

Interviewer $I_{1}$ kann in seiner Teilstichprobe nur eine der 3 folgenden Kontingenztafeln realisieren:
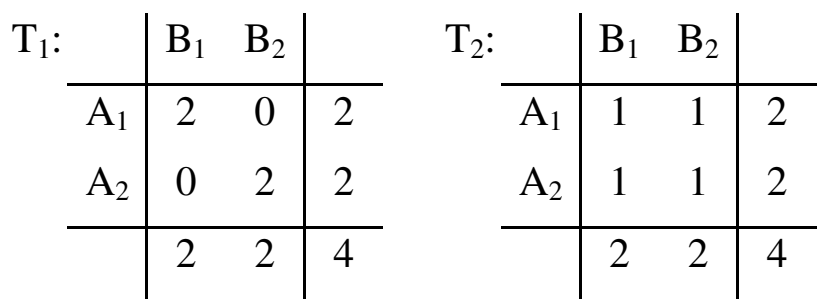

\begin{tabular}{c|cc|c}
$\mathrm{T}_{3}:$ & $\mathrm{B}_{1}$ & $\mathrm{~B}_{2}$ & \\
\hline $\mathrm{A}_{1}$ & 0 & 2 & 2 \\
$\mathrm{~A}_{2}$ & 2 & 0 & 2 \\
\hline & 2 & 2 & 4
\end{tabular}

Die Eintreffwahrscheinlichkeiten für diese Kontingenztafeln betragen: $\operatorname{Pr}\left(\mathrm{T}_{1}\right)=0,338$, $\operatorname{Pr}\left(\mathrm{T}_{2}\right)=0,550$ und $\operatorname{Pr}\left(\mathrm{T}_{3}\right)=0,112$.

Mit (6) errechnen sich folgende Erwartungswerte $\mathrm{p}_{\mathrm{ij}}$ für die kombinierten Quoten in dieser von $\mathrm{I}_{1}$ erhobenen Teilstichprobe:

\begin{tabular}{c|cc|c}
$\mathrm{E}_{\mathrm{I}_{1}} \mathrm{p}_{\mathrm{ij}}:$ & $\mathrm{B}_{1}$ & $\mathrm{~B}_{2}$ & \\
\hline $\mathrm{A}_{1}$ & 0,3065 & 0,1935 & 0,5 \\
$\mathrm{~A}_{2}$ & 0,1935 & 0,3065 & 0,5 \\
\hline & 0,5 & 0,5 & 1
\end{tabular}

In dieser Teilstichprobe wird also im Durchschnitt die Häufigkeit des Auftretens der Ausprägung $A_{1} B_{1}$ stark überschätzt und daher die Verteilung der Stichprobe auf dem Untersuchungsmerkmal $\mathbf{X}$ innerhalb dieser Schicht zu stark für die $\mathrm{zu}$ schätzende Verteilung der Grundgesamtheit auf $\mathbf{X}$ gewichtet. Das Auftreten der Ausprägung $\mathrm{A}_{2} \mathrm{~B}_{2}$ wird hingegen deutlich unterschätzt. $\mathrm{I}_{2}$ bringt mit seiner Teilstichprobe 4 Objekte mit Ausprägung $\mathrm{A}_{2} \mathrm{~B}_{2}$ in die Gesamtstichprobe ein und wir erhalten insgesamt folgende Erwartungswerte für die kombinierten Quoten in der Grundgesamtheit:

\begin{tabular}{|c|c|c|c|}
\hline \multirow{2}{*}{$\mathrm{E}_{\mathrm{ges}} \mathrm{p}_{\mathrm{ij}}$} & \multicolumn{2}{|c|}{ B } & \\
\hline & $\mathrm{B}_{1}$ & $\mathrm{~B}_{2}$ & \\
\hline \multirow{3}{*}{$\mathrm{A}_{2}$} & 0,15325 & 0,09675 & 0,25 \\
\hline & 0,09675 & 0,65325 & 0,75 \\
\hline & 0,25 & 0,75 & 1 \\
\hline
\end{tabular}

Es entsteht somit eine hinsichtlich der eindimensionalen Merkmale A und B exakt repräsentative Stichprobe vom Umfang $\mathrm{n}=8$, die hinsichtlich der zweidimensionalen Verteilung der Stichprobe auf dem Merkmal AB stark verzerrt ist. 
Die in der Praxis verwendeten Quotenanweisungen an die Interviewer (siehe Abschnitt 3 dieses Aufsatzes) zeigen, daß die Vierfeldertafeln dieses Abschnitts keine praxisfernen Beschränkungen darstellen. Auch wird in der Praxis keinerlei Rücksicht auf die exakte Repräsentativität der Verteilung der Teilstichproben (der Interviewer) auf den einzelnen Quotenmerkmalen genommen und ausschließlich auf das Einhalten dieser in der Gesamtstichprobe geachtet. Damit entstehen - wie Beispiel 7 zeigt - noch wesentlich größere Verzerrungen hinsichtlich der Verteilung der Stichprobe auf dem kombinierten Quotenmerkmal (vgl. dazu auch Quatember, 1996b, S.106) und damit auch hinsichtlich des Schätzers $\overline{\mathrm{x}}_{\mathrm{RQ}}$ (3) als bei exakter Repräsentativität innerhalb jeder der Teilstichproben (siehe oben).

Eine Vermutung bezüglich der Schätzergenauigkeit, der zweiten zu überprüfenden statistischen Eigenschaft des Schätzers $\overline{\mathrm{x}}_{\mathrm{RQ}}$ (3) bei Auswahlen nach Randquoten, bezieht sich auf die diesbezügliche intuitive Einordnung der Konzeption der Auswahl nach Randquoten unter Urnenmodellbedingungen zwischen die Auswahl nach kombinierten Quoten unter Urnenmodellbedingungen einerseits und die uneingeschränkte Zufallsauswahl andererseits. Quatember (1996b) zeigt, daß diese Vermutung der Überprüfung durch exakte numerische Berechnung von Schätzervarianzen und durch deren empirische Bestimmung mit einem $\mathrm{zu}$ diesem Zweck entwickelten Simulationsprogramm nicht standhält: Die Schätzervarianz ist in Auswahlen nach Randquoten unter Urnenmodellbedingungen tatsächlich durch jene der Auswahlen nach kombinierten Quoten nach unten hin begrenzt, sie kann jedoch jene der uneingeschränkten Zufallsauswahlen nach oben hin noch übersteigen. Die Genauigkeit ist dabei (unter anderem) eine Funktion der Einzelassoziationen zwischen den Quotenmerkmalen $\mathbf{A}, \mathbf{B}, \mathbf{C}, \ldots$ und dem Untersuchungsmerkmal $\mathbf{X}$ - im Unterschied zur gar nicht von solchen Zusammenhängen abhängigen Genauigkeit in uneingeschränkten Zufallsauswahlen und zur alleine vom Zusammenhang zwischen dem kombinierten Quotenmerkmal ABC... und dem Untersuchungsmerkmal $\mathbf{X}$ abhängigen Genauigkeit von Auswahlen nach kombinierten Quoten (vgl. Quatember, 1994). Die Möglichkeit eines negativen „Quotierungseffektes“ entsteht durch die wegen der Vorgabe der Randquoten (im Vergleich zu einer uneingeschränkten Zufallsauswahl) hervorgerufene Einschränkung bei den möglichen Verteilungen der Stichprobe auf den Quotenmerkmalen (siehe etwa Beispiel 1). Diese kann, so sich die Schichtmittelwerte $\mu_{\mathrm{ij}}$ von $\mathbf{X}$ in den durch die Vorgabe der Randquoten überschätzten Kombinationen von ABC... wesentlich von denjenigen in den unterschätzten Kombinationen unterscheiden, zu einer Streuung des

Stichprobenmittelwertes $\mathrm{x}_{\mathrm{RQ}}$ führen, die jene des Stichprobenmittelwertes $\overline{\mathrm{x}}_{\mathrm{Z}}$ aus uneingeschränkten Zufallsauswahlen übersteigt (vgl. Quatember, 1996b, S. 73ff).

\section{Schlußfolgerungen}

Es wurde gezeigt, daß eine Auswahl nach kombinierten Quoten unter Urnenmodellbedingungen einer nach einem mehrdimensionalen Merkmal geschichteten Zufallsauswahl entspricht. Für die Praxis stellt sich bei Verwendung dieses Verfahrens allenfalls ein Rundungsproblem ein, wenn nämlich ein relativ geringer Stichprobenumfang proportional auf die Schichten allokiert werden soll. So hat z.B. das sowohl in 
Österreich als auch in Deutschland gebräuchliche Quotenmerkmal Wohnbundesland und Geschlecht und Alter und Berufstätigkeit und Berufsgruppe (vgl. Noelle, 1963, S. 133; Quatember, 1996b, S. 100) in Österreich 720 Ausprägungen.

Die Auswahl nach Randquoten ist selbst unter Urnenmodellbedingungen unbefriedigend. Es erfüllt sich die Hoffnung nicht, daß die exakte Repräsentativität der Stichprobe hinsichtlich mehrerer Randmerkmale auch die Repräsentativität der Stichprobe hinsichtlich der Untersuchungsmerkmale nach sich zieht, denn die Schätzer für die kombinierten Quoten der verwendeten Quotenmerkmale sind verzerrt. Die angebbaren Bedingungen, unter denen eine erwartungstreue Schätzung der kombinierten Quoten doch eintreten kann, sind die Unabhängigkeit der verwendeten $\mathrm{m}$ Randmerkmale und die Gleichverteilung eines Teils dieser Merkmale.

Die Merkmale Wohnbundesland und Geschlecht werden in der Praxis kombiniert vorgegeben, so daß die Stichprobe hinsichtlich dieses Merkmals exakt repräsentativ ist. Die Quotenanweisungen für die Interviewer enthalten dann innerhalb dieser fix vorgegebenen Teilgesamtheiten die Randquoten des eindimensionalen Merkmals Alter und des zweidimensionalen Merkmals Berufstätigkeit und Berufsgruppe (Abbildung 4).

\begin{tabular}{|c|c|c|c|c|c|c|c|c|c|}
\hline & \multicolumn{8}{|c|}{ Berufstätigkeit und Berufsgruppe } & \\
\hline & \multicolumn{4}{|c|}{ Berufstätigkeit $=$ ja } & \multicolumn{4}{|c|}{ Berufstätigkeit=nein } & \\
\hline & Bgr.1 & Bgr.2 & Bgr.3 & Bgr.4 & Bgr.1 & Bgr.2 & Bgr.3 & Bgr.4 & \\
\hline $15-29$ & $\mathrm{p}_{1,11}$ & $\mathrm{p}_{1,12}$ & $\ldots$ & & & & & & $\pi_{1, . .}$ \\
\hline $30-39$ & $\mathrm{p}_{2,11}$ & & & & & & & & $\pi_{2, \ldots}$ \\
\hline \multirow[t]{4}{*}{ Alter } & $\vdots$ & & & & & & & & : \\
\hline & & & & & & & & & \\
\hline & & & & & & & & & \\
\hline & $\pi_{., 11}$ & $\pi_{., 12}$ & $\ldots$ & & & & & & 1 \\
\hline
\end{tabular}

Abbildung 4: Die Verteilung der Gesamtstichprobe auf den Quotenmerkmalen bei einer Auswahl nach Randquoten und Verwendung der Quotenmerkmale Alter und Berufstätigkeit und Berufsgruppe innerhalb des durch kombinierte Quotierung vorgegebenen Merkmals Wohnbundesland und Geschlecht.

(Bgr.1: Landwirte, Landarbeiter; Bgr.2: Arbeiter; Bgr.3: Angestellte, Beamte; Bgr.4: Selbständige, freie Berufe)

Diese beiden Merkmale sind sicherlich nicht statistisch unabhängig voneinander, gehört doch etwa zu den Nichtberufstätigen, deren Berufsgruppe bei Rentnern durch den früheren Beruf, bei Hausfrauen, Schülern etc. durch den Beruf des Ernährers, wie es in den Quotenanweisungsbögen der Markt- und Meinungsforschungsinstitute heißt (vgl. ebd., S. 100), bestimmt wird, ein Personenkreis, dessen Verteilung auf dem Merkmal Alter keinesfalls auch nur annähernd jener der Gesamtbevölkerung auf diesem Merkmal entspricht. 
Auch ist die Verteilung der Grundgesamtheit auf beiden Randmerkmalen weit von einer Gleichverteilung entfernt. Dies ist beim zweidimensionalen Berufsmerkmal wegen der höheren Zahl an Berufstätigen als an Nichtberufstätigen auch tatsächlich nicht möglich. Beim Merkmal Alter ließe sich durch geeignete Intervalleinteilung jedoch sehr leicht eine Gleichverteilung der Grundgesamtheit auf die einzelnen Intervalle herstellen. Die Einteilung, die in der Praxis vorgenommen wird (siehe Abbildung 4), ist jedoch keine, die eine Gleichverteilung erzeugt (Tabelle 2).

Tabelle 2: Verteilung der österreichischen Bevölkerung (15 Jahre und älter) auf dem Merkmal Alter (Intervalle nach Abbildung 4; Daten aus: Statistisches Jahrbuch der Republik Österreich 1996, Hrsg.: Österreichisches Statistisches Zentralamt, S.22).

\begin{tabular}{cc}
\hline Altersklasse & Anteil \\
\hline $15-29$ & 0,224 \\
$30-39$ & 0,211 \\
$40-49$ & 0,164 \\
$50-59$ & 0,149 \\
60 J. u. älter & 0,251 \\
\hline
\end{tabular}

Die bedingten Verteilungen jener Teilgesamtheiten, die durch das Wohnbundesland und das Geschlecht der Befragten gebildet werden, auf dem Merkmal Alter weichen von der in Tabelle 2 dargestellten Verteilung der (über 14-jährigen) Gesamtbevölkerung leicht ab. Doch auch diese Teilgesamtheiten sind auf dem Merkmal Alter bei dieser Intervalleinteilung nicht gleichverteilt.

Eine geringere statistische Abhängigkeit zwischen den vorgegebenen Quotenmerkmalen könnte erreicht werden, wenn anstelle des zweidimensionalen Berufsmerkmals (siehe Abbildung 4) nur das eindimensionale Merkmal Berufsgruppe verwendet würde. Dies würde unter Urnenmodellbedingungen gemeinsam mit einer Veränderung in der Intervalleinteilung beim Merkmal Alter eine Verzerrungsminderung hinsichtlich der kombinierten Quoten dieser Merkmale bewirken, ohne daß die Grundgesamtheit auch auf dem Merkmal Berufsgruppe (Tabelle 3) gleichverteilt sein müßte.

Tabelle 3: Verteilung der berufstätigen österreichischen Bevölkerung auf dem in Abbildung 2 definierten Merkmal Berufsgruppe (Daten aus: Statistisches Jahrbuch der Republik Österreich 1996, Hrsg.: Österreichisches Statistisches Zentralamt, S. 131).

\begin{tabular}{cc}
\hline Berufsgruppe & Anteil \\
\hline 1 & 0,063 \\
2 & 0,375 \\
3 & 0,484 \\
4 & 0,078 \\
\hline
\end{tabular}

Aus der Sicht der Praktiker mag nun eingewendet werden, daß es besser sei, auch die Verteilung der Grundgesamtheit auf dem Merkmal Berufstätigkeit durch Vorgabe der 
Randquoten $\mathrm{zu}$ kontrollieren, da bei unsorgfältig arbeitenden Interviewern die Verteilung der Stichprobe gerade auf diesem Merkmal von der tatsächlichen Verteilung stark abweichen kann. Dem ist entgegenzuhalten, daß doch gerade das von den Praktikern entworfene Bedingungssystem (siehe Abschnitt 1) eine zufallsauswahlähnliche Objekteselektion innerhalb der vorgegebenen Quoten durch die Interviewer zum Ziel hat. Eine besondere Instruktion der Interviewer im Rahmen der Interviewerschulung könnte also auch dieser Verzerrungsmöglichkeit durchaus entgegenwirken.

Doch wie man es auch betrachtet, die Ergebnisse der Untersuchung der Funktionstauglichkeit der Randquotenauswahlen bedeuten, daß eine konkrete Varianzschätzung selbst unter Urnenmodellbedingungen nicht erfolgen kann. Daher sind etwa Signifikanztests oder die Bestimmung von Konfidenzintervallen oder multivariate Verfahren bei der statistischen Verarbeitung von Daten, die mit dieser Auswahltechnik erhoben wurden, nicht zugängliche Methoden. Quatember (1996b) zeigte, daß die ersatzweise Verwendung der Varianz uneingeschränkter Zufallsauswahlen - wie dies z.B. bei der Verwendung statistischer Programmpakete bei der Datenanalyse automatisch der Fall ist - nicht erlaubt ist.

Schließlich weist diese Auswahltechnik auch noch das Problem der Aufteilung der Gesamtstichprobe auf die einzelnen Interviewer auf, dem sich Quatember (1996b) ebenfalls widmet.

Ein wesentlicher Vorteil dieser Auswahltechnik bleibt schließlich allen Bedenken zum Trotz unbestritten: der geringe Zeitaufwand. Sind also deskriptive Daten zu bestimmten Themen zu erheben und müssen die Ergebnisse „praktisch brauchbar“, aber nicht ,statistisch genau“ sein, dann sind auch Quotenstichproben nach Randquoten unter Voraussetzung verantwortungsbewußter, gut geschulter Interviewer eine taugliche Möglichkeit zur Gewinnung dieser Informationen. Doch „,man denke stets daran, daß Normen aus einer wirklich repräsentativen Stichprobe von wenigen $100 \mathrm{Pbn}$ wertvoller sind als solche von mehreren $1000 \mathrm{Pbn}$ aus einer nicht repräsentativen Stichprobe“ (Lienert, 1967, S. 318). Dem ist nichts hinzuzufügen.

\section{Literatur}

D.R. Bellhouse. A Brief History of Random Sampling Methods. In: P.R. Krishnaiah, C. R. Rao, Herausgeber, Handbook of Statistics, Vol. 6, 1-14. Elsevier Science Publishers B.V., Amsterdam, 1988.

J. Bortz und N. Döring. Forschungsmethoden und Evaluation. Springer, Berlin, 2. Auflage, 1995.

S. Kotz and N.L. Johnson. Encyclopedia of Statistical Sciences, Vol. 7. John Wiley \& Sons, New York, 1986.

W. Kruskal and F. Mosteller. Representative Sampling, IV: The History of the Concept in Statistics, 1895-1939. International Statistical Review, 48, 169-195, 1980. 
G.A. Lienert. Testaufbau und Testanalyse. Verlag Julius Beltz, Weinheim/Berlin, 2. Auflage, 1967.

E. Noelle. Umfragen in der Massengesellschaft. Rowohlt, Hamburg, 1963.

A. Quatember. Der Schichtungseffekt bei der Erhebung von Anteilen - Der Genauigkeitsgewinn gemessen am Grad des Zusammenhanges zwischen Untersuchungs- und Schichtmerkmal. Allgemeines Statistisches Archiv, 78, 318-322, 1994.

A. Quatember. Das Problem mit dem Begriff Repräsentativität. Allgemeines Statistisches Archiv, 80, 236-241, 1996.

A. Quatember. Das Quotenverfahren. Schriften der Johannes-Kepler-Universität Linz, Reihe B - Wirtschafts- und Sozialwissenschaften. Universitätsverlag Rudolf Trauner, Linz, 1996.

A. Quatember. Schichten mit geschätzten Schichtgrößen. Österreichische Zeitschrift für Statistik, 25. Jahrgang, Heft 1, 43-58, 1996.

G. Schmidtchen. Die repräsentative Quotenauswahl. Bericht über ein Quota-RandomExperiment des Instituts für Demoskopie Allensbach. Institut für Demoskopie Allensbach, 1961.

H. Stenger. Stichproben. Physica-Verlag, Heidelberg, Wien, 1986.

Adresse des Autors:

Dr. Andreas Quatember

Institut für Angewandte Statistik

Johannes-Kepler-Universität Linz

Altenbergerstr. 69, A-4040 LINZ

Tel.: (0732) 2468/586

Fax: (0732) 2468/9846

e-mail: andreas.quatember@jk.uni-linz.ac.at 